\title{
ALFABETIZACIÓN EN SALUD EN EL SISTEMA SANITARIO: OBJETIVOS Y POSIBLES ACTUACIONES
}

\author{
HEALTH LITERACY IN THE HEALTH SYSTEM: GOALS AND ACTIONS
}

Ma Dolores Navarro Rubio ${ }^{1, a}$

${ }^{1}$ Hospital Sant Joan de Déu, Barcelona

a mariadolors.navarro@gmail.com

Recibido: 20/09/2021; Aceptado: 06/10/2021

\section{Resumen}

El nivel de alfabetización en salud de los pacientes se ha convertido en un tema clave para los profesionales y las organizaciones sanitarias, ya que les permite valorar las habilidades y competencias de los pacientes al utilizar la información sobre su propia enfermedad y sobre cómo pueden actuar en su abordaje y manejo. Numerosos estudios han mostrado cómo las personas que presentan un bajo nivel de alfabetización sanitaria pueden tener dificultades para entender las indicaciones de los profesionales sanitarios y presentan un peor estado de salud y calidad de vida.

Se ha de preparar a los propios profesionales en estos aspectos de alfabetización en salud que les permita mejorar sus habilidades comunicativas y empáticas a la hora de comunicarse con pacientes y familiares. Los profesionales sanitarios han de ser conscientes de que su manera de comunicar y de dirigirse a los pacientes influirá en cómo éstos pueden responder ante su situación, en cuanto a su autocuidado y abordaje de la enfermedad o respecto al seguimiento de las recomendaciones o cumplimiento terapéutico. De la misma forma, es necesario un enfoque estratégico de la propia institución. Así pues, la dirección del centro sanitario debe contemplar también la incorporación del paciente en la toma de decisiones.

La alfabetización en salud de los pacientes, por lo tanto, mejorará mucho más si este abordaje se realiza de una manera integral, teniendo en cuenta a los pacientes y familiares, pero también considerando las habilidades comunicativas de los profesionales y las directrices de la organización. Además, para que las intervenciones en la mejora del nivel de alfabetización sanitaria de la población sean efectivas, las administraciones, los sistemas sanitarios en su globalidad, han de incorporar entre sus directrices, elementos que favorezcan la implementación de dichas acciones.

Palabras clave: Alfabetización en salud; participación del paciente; profesionales sanitarios; sistema sanitario

\section{Abstract}

The level of patients' health literacy has become a key issue for health professionals and organizations, as it allows them to assess the skills and competencies of patients when using information about their own disease and how they can manage it. Numerous studies have shown how people with a low level of health literacy may have difficulties understanding the instructions of health professionals and have a worse health status and quality of life.

The professionals themselves must be trained in these aspects of health literacy in order to improve their communication and empathic skills when communicating with patients and families. Health professionals must be aware that their way of communicating and addressing patients will influence 
how they can respond to their situation, in terms of their self-care and approach to the disease, or regarding the follow-up of recommendations or therapeutic compliance. In the same way, a strategic approach from the institution itself is necessary. Thus, the management of the health center must also consider the incorporation of the patient in decision-making.

The health literacy of patients, therefore, will improve much more if this approach is carried out in a comprehensive way, taking into account patients and families, but also considering the communication skills of the professionals and the guidelines of the organization. In addition, for interventions to improve the population's health literacy level to be effective, the administrations, the health systems as a whole, have to incorporate among their guidelines, elements that favor the implementation of said actions.

Keywords: Health literacy; patient participation; health professionals; health system.

\section{INTRODUCCIÓN}

La alfabetización en salud se refiere a la capacidad de las personas para acceder a información de calidad sobre salud y saber aplicar dicha información en el autocuidado, tomando las decisiones apropiadas y haciendo un uso correcto de los servicios sanitarios (Neilsen-Bohlman et al., 2004). Este concepto es importante para los profesionales y las organizaciones sanitarias en el momento de valorar las habilidades y competencias de los pacientes al utilizar la información sobre su propia enfermedad y sobre cómo pueden actuar en su abordaje y manejo.

En las últimas décadas se han realizado gran número de estudios que han mostrado cómo las personas que presentan un bajo nivel de alfabetización sanitaria pueden tener dificultades para entender las indicaciones de los profesionales sanitarios, las pautas terapéuticas a seguir, los consejos preventivos o de promoción de la salud y calidad de vida o los informes de alta hospitalaria, entre otra mucha información que suelen recibir. Se ha descrito también que niveles bajos de alfabetización sanitaria están relacionados con un peor estado de salud y una mortalidad más elevada entre determinados grupos de la población, provocando incluso un mayor gasto en el sistema sanitario (Howard et al., 2005; Berkman et al., 2011).

Los primeros estudios que se realizaron sobre la alfabetización en salud se centraron en el papel del paciente en la adquisición de un buen nivel de conocimiento, poniendo énfasis en el paciente o en sus familiares y cuidadores. Es decir, se daba a los propios pacientes la responsabilidad de acceder a la información, interpretarla y seguir las recomendaciones adecuadas para poder cuidar de su salud y hacer un abordaje correcto de su enfermedad. Sin embargo, estudios posteriores pusieron de manifiesto que la alfabetización en salud es un concepto mucho más amplio que engloba también a otros actores de la atención y del sistema sanitario. Así pues, si en un primer momento se puso la atención sobre el paciente y sus características, con el paso del tiempo se ha visto que son tres los elementos necesarios para conseguir un buen nivel de alfabetización en salud: los pacientes y familiares, los profesionales y las instituciones sanitarias (Navarro Rubio et al., 2016).

De esta forma, los profesionales sanitarios han de ser conscientes de que su manera de comunicar y de dirigirse a los pacientes influirá en cómo éstos pueden responder ante su situación, en cuanto a su autocuidado y abordaje de la enfermedad o respecto al seguimiento de las recomendaciones o cumplimiento terapéutico. En este sentido, se ha de formar a los propios profesionales en estos aspectos de alfabetización en salud que les permita mejorar sus habilidades comunicativas y empáticas a la hora de comunicarse con pacientes y familiares. 
Igualmente, no se podría avanzar en temas de participación del paciente o de la atención sanitaria basada en la experiencia del paciente sin un enfoque estratégico de la propia institución. Así pues, la dirección del centro sanitario debe contemplar también la incorporación del paciente en la toma de decisiones, bien sea a nivel individual, teniendo cuidado de la propia salud o a nivel colectivo, en la mejora de servicios y procesos o bien, representando a otros pacientes.

La alfabetización en salud de los pacientes, por lo tanto, mejorará mucho más si este abordaje se realiza de una manera integral, teniendo en cuenta a los pacientes y familiares, pero también considerando las habilidades comunicativas de los profesionales y las directrices de la organización. De la misma forma, para que las intervenciones en la mejora del nivel de alfabetización sanitaria de la población sean efectivas, las administraciones, los sistemas sanitarios en su globalidad, han de incorporar entre sus directrices, elementos que favorezcan la implementación de dichas acciones.

\section{LOS PACIENTES Y SUS FAMILIAS O CUIDADORES}

La sociedad actual se encuentra inmersa en un momento de gran cambio en todas sus esferas, tanto demográfica, económica, cultural o tecnológica como también sanitaria. La reciente pandemia por COVID-19 es un claro ejemplo de cambio y adaptación en diferentes ámbitos. Y, en general, la ciudadanía cuenta en la actualidad con un mayor nivel formativo y de participación, pudiendo acceder de forma rápida a gran volumen de información sobre salud (Navarro Rubio et al., 2008).

A su vez, se ha producido un cambio en el tipo de relación que se establece entre el profesional sanitario y el paciente, pasando de una relación paternalista y dependiente a una relación en la que el paciente adquiere un papel más activo a la hora de hablar con el profesional de su problema de salud y tomar decisiones conjuntamente. La relación establecida entre el profesional y el paciente en estos casos tiende a volverse más colaborativa y simétrica para llegar a un proceso deliberativo de toma de decisiones compartidas (Navarro Rubio, 2014; Montori, 2017).

Sin embargo, la aparición de este nuevo modelo de paciente más activo no significa que exista un aumento general de la alfabetización sanitaria en la población (Neilsen-Bohlman et al, 2004; Navarro Rubio et al., 2016). Más bien al contrario, los pacientes perciben la asimetría de información y conocimiento respecto a los profesionales como un elemento que dificulta su participación en los procesos de toma de decisiones que afectan a su salud.

La comunicación y la transmisión de información, ya sea oral o escrita, en papel u online, ejerce un impacto importante en la mejora de la atención sanitaria. Situaciones como el incumplimiento terapéutico de los enfermos crónicos en atención primaria, el control inadecuado de los pacientes polimedicados o la elevada prevalencia de estilos de vida poco saludables pueden ser ejemplos de situaciones en las que influye el déficit de alfabetización en salud (Navarro Rubio et al., 2016).

Actualmente, el nivel de alfabetización en salud de la población es un tema de gran interés, tanto para la tarea asistencial, como para la salud pública y la gestión sanitaria. En un estudio sobre el nivel de alfabetización en salud en la Unión Europea, más de la mitad de los encuestados españoles presentaban un nivel limitado de alfabetización en salud (Sørensen et al., 2011). Esta misma situación se corroboró en un estudio de la OCDE sobre las competencias de los adultos en España (OCDE, 2013). Este hecho, como se ha comentado, es relevante ya que las personas con una baja alfabetización suelen obtener peores resultados en salud en 
comparación a aquellas con un nivel adecuado. Así, por ejemplo, presentan mayor riesgo de hospitalizaciones, mayor número de admisiones en servicios de urgencias, peor adherencia a los tratamientos, mayor mortalidad o menor nivel de bienestar y calidad de vida (Berkman et al., 2011).

Es necesario, por lo tanto, valorar el papel que ejercen también los profesionales sanitarios en la mejora de las competencias en salud de los pacientes.

\section{EL PAPEL DE LOS PROFESIONALES SANITARIOS}

Tanto los profesionales como los sistemas sanitarios han de hacer frente a este cambio que se está produciendo en diferentes áreas de la sociedad. Los profesionales se han de capacitar para atender, de la mejor forma posible, las nuevas necesidades de la población a la que atienden. Esta sociedad exige cada vez más a los profesionales sanitarios que den respuesta a la complejidad del proceso asistencial y de las intervenciones realizadas, la incorporación de nuevas tecnologías o el coste asociado que todo ello conlleva; aparte de incorporar los valores, preferencias y experiencia del paciente.

Además, como ya se ha comentado, el cambio producido en el perfil del paciente también implica un cambio importante en la relación con los profesionales sanitarios, ya que supone el paso de un modelo paternalista a otro deliberativo y basado en la toma de decisiones compartidas. El intercambio de información y la comunicación que se produce durante la consulta o visita médica representa uno de los momentos más importantes de la relación entre profesional y paciente. Este encuentro es la base del sentimiento de confianza necesario para el tratamiento y seguimiento de cada paciente.

De esta forma, durante la visita o encuentro médico, el profesional realizará preguntas al paciente sobre lo que le ocurre y desde cuándo, contestará a sus preguntas y le dará información sobre un posible diagnóstico, tratamiento o pasos a seguir. Igualmente, se espera que el paciente sea capaz de transmitir lo que le ocurre y de contestar a cualquier información relacionada con lo que le pasa. El paciente ha de ser capaz también de recordar lo que se comente en la consulta para poder seguir las indicaciones realizadas por el profesional de forma correcta. La comprensión de la información sobre salud es indispensable para que el encuentro médico sea efectivo. Es lo que se conoce como "Modelo conceptual del aprendizaje en salud" (Wolf et al., 2009).

Pero, además de los aspectos generales que favorecen una buena relación, como podrían ser una actitud abierta, positiva, habilidades comunicativas o la percepción que se tiene del otro, el grado de satisfacción con la consulta se verá afectado por aspectos como establecer un buen clima de diálogo antes de entrar en temas concretos de diagnóstico o tratamiento, apoyar y valorar las acciones o actitudes positivas del paciente respecto a su situación, animarlo a continuar, valorar los pequeños logros o avances y mostrar interés por sus comentarios, dudas o preocupaciones. El lenguaje corporal también es importante a este nivel, mostrando una actitud atenta e interesada por el paciente.

Para conseguir este nivel de relación es necesario que los profesionales sanitarios, así como los pacientes, puedan prepararse para ese encuentro (la consulta o visita médica) tanto en habilidades comunicativas como en la gestión del tiempo y de la complejidad que alcanzar un buen estado de salud conlleva.

De esta forma, la alfabetización en salud no se centra sólo en las características de los pacientes. Es necesario también que los profesionales adquieran unas características específicas en este sentido; unas competencias y habilidades que les permitan comunicarse con 
efectividad y empatía hacia el paciente. Es preciso reducir las barreras que existen en la relación profesional-paciente que hacen que éste último no entienda la información que se le transmite. Los profesionales han de facilitar al paciente el poder llevar a cabo su plan de cuidados. Han de asegurarse de que transmiten una información clara y concisa que el paciente pueda entender y han de crear un clima tal que permita al paciente intercambiar opiniones y hacer preguntas si algo no le ha quedado suficientemente claro o le plantea dudas.

La terminología médica suele ser compleja, por lo que el uso de jerga o de tecnicismos que no se explican con claridad al paciente pueden suponer una barrera a la hora de entender esa información. De esta forma, se han desarrollado diferentes estrategias para simplificar el vocabulario médico sin que pierda rigor científico (Koch-Weser et al., 2009). La valoración de estos aspectos por parte del profesional sanitario en el entorno clínico está documentada por diversos autores, ya que permite adecuar el contenido, la complejidad de los mensajes y las intervenciones dirigidas a los pacientes (Neilsen-Bohlman et al., 2004; Lai et al., 2013; Heijmans et al., 2015). Es necesario desarrollar estrategias de información y documentación que garanticen la accesibilidad a contenidos de salud de calidad acreditada en un lenguaje comprensible, además de promover la mejora de las capacidades y habilidades de los pacientes, familiares y cuidadores mediante la identificación y formación de competencias específicas (Navarro Rubio et al., 2018).

Por este motivo, en los últimos años se han desarrollado diferentes instrumentos de valoración de la alfabetización de sanitaria. Estos instrumentos, aunque muy útiles, han presentado algunas limitaciones, como el tiempo elevado de administración, la poca precisión de algunos resultados o resultar incómodos en las personas examinadas (Davis et al., 1993; Parker et al., 1995; Haun et al., 2012). Estudios más recientes realizados por investigadores españoles han desarrollado escalas de valoración de las competencias en salud de los pacientes, adaptadas en concreto a la atención primaria de salud (González de Paz et al., 2019; Navarro Rubio et al., 2019).

Otros estudios han puesto de manifiesto cuáles son algunos de los errores más frecuentes en la comunicación entre profesionales sanitarios y paciente, como, por ejemplo, preguntarle al paciente directamente si ha entendido la información que se le ha dado. Realizar este tipo de preguntas directas puede inhibir el diálogo, posicionar al paciente en una situación de dificultad, sin promover la exposición de dudas o dificultades (Kutner et al., 2006; Weiss, 2007). El paciente, además, puede sentirse incómodo y no atreverse a aceptar que no ha entendido la información. En estos casos se aconseja utilizar la técnica del "teach back" mediante la que el profesional preguntaría al paciente si se ha explicado bien, poniendo la responsabilidad de la claridad de la información en el profesional en vez de en el paciente (Rudd, 2010; Speros, 2011).

Otra de las principales recomendaciones a la hora de escribir cualquier mensaje dirigido a los pacientes, cuidadores o ciudadanos en general, es el empleo de un lenguaje cotidiano, cercano y que evite al máximo la utilización de terminología técnica o jerga. Este hecho, al contrario de lo que podría parecer, no desacredita su validez científica, ni la de los profesionales que participaron en su elaboración (Kimble, 2002).

En ocasiones, puede ocurrir también que las personas que han alcanzado un nivel de alfabetización óptimo presentan igualmente dificultades para manejarse en el entorno sanitario, ya que no cuentan con las competencias específicas para responder a esa situación (Nutbeam, 2000). Otros autores han estudiado las respuestas que las personas que presentan bajas competencias en salud suelen dar como excusa en el momento en que tienen dificultades para comprender una información tales como decir que olvidaron las gafas, que lo leerán después o que estos temas se los lleva su hijo/a (Weiss, 2007). 
Los profesionales de la salud deben comprender, aceptar y motivar este nuevo rol en el paciente para tener un modelo real y eficaz de participación y empoderamiento del paciente en la toma de decisiones. También es necesario educar a los profesionales en el nuevo rol del paciente en la toma de decisiones para promover la existencia de una relación de confianza y cooperación entre ellos (Halvorsen et al., 2020). Existen algunos estudios que muestran cómo formar profesionales en aspectos específicos de la atención integral (Stiggelbout et al., 2012); así como en las acciones a realizar para asegurar que la comunicación con los pacientes sea efectiva y acorde con sus necesidades y expectativas (Patak et al., 2009; Yen y Leasure, 2019).

Mantener esta relación deliberativa entre el paciente y el profesional significa que los pacientes tienen la información necesaria adaptada a sus necesidades que les permite hablar con confianza sobre sus preferencias y tomar decisiones informadas. Al mismo tiempo, los profesionales de la salud se sienten útiles acompañando a los pacientes en el proceso de toma de decisiones. Finalmente, para lograr esta relación deliberativa, es fundamental que tanto el paciente como el profesional de la salud estén preparados y capacitados para el establecimiento de este nuevo tipo de relación.

\section{LAS ORGANIZACIONES Y EL SISTEMA SANITARIO}

El objetivo general del estudio de la alfabetización sanitaria es ayudar a las poblaciones a adquirir un mejor control de su salud. Mediante la difusión de los resultados de estudios e intervenciones, pacientes, profesionales e instituciones pueden reorganizar sus acciones conjuntamente para mejorar la atención sanitaria, a toda la población, independientemente del nivel de alfabetización en salud que presenten (Paasche-Orlow et al., 2018).

La incorporación de los pacientes en los diferentes entornos sanitarios se ha de realizar de forma responsable, sin que suponga una moda pasajera. Los pacientes han de poder participar en los temas y en los momentos en los que realmente su presencia sea necesaria. En este sentido, las organizaciones sanitarias deben disminuir la responsabilidad que normalmente se pone en los pacientes. Así, se conoce como "alfabetización en salud de la organización" al grado en que se implementan políticas, prácticas y sistemas de manera que se facilite la navegación de las personas por el entorno sanitario, de manera que se entienda y se utilice la información sobre salud (Brach et al., 2012; Brega et al., 2019).

Dentro de esta alfabetización en salud de la organización, existen seis factores que se interpretan como esenciales: favorecer la comunicación con los pacientes, mejorar el acceso y la navegación del paciente por la organización, facilitar la incorporación del paciente en la mejora de los servicios, establecer grupos de trabajo en alfabetización en salud, crear una cultura y una infraestructura en la organización que favorezca la alfabetización en salud y dar respuesta las necesidades del paciente. Estos conceptos pueden integrarse en áreas de trabajo en las que intervenir para disminuir la carga que representa para el paciente, por ejemplo, a nivel de comunicación, facilidad de navegación y apoyo a la participación (Brega et al., 2019).

Así, toda organización sanitaria debe contar con unos atributos para poder caracterizarse como una organización orientada a la alfabetización en salud. Entre estos atributos se podría destacar: integrar los aspectos relacionados con la alfabetización en salud en la planificación y evaluación de acciones de mejora, formar a sus profesionales en esta área de conocimiento, analizar las necesidades de información y participación de la población a la que se dirige o favorecer el acceso a información de calidad, entre otros atributos (Tabla 1) (Brach et al., 2012). 
Tabla 1. Atributos en alfabetización en salud de las organizaciones sanitarias. Fuente: adaptado de Brach et al. (2012).

\begin{tabular}{|l|}
\hline \multicolumn{1}{|c|}{ ATRIBUTOS } \\
\hline $\begin{array}{l}\text { Capacidad de liderazgo para integrar la alfabetización en salud en la misión, estructura y } \\
\text { operaciones de la organización. }\end{array}$ \\
\hline $\begin{array}{l}\text { Incorporación de la alfabetización en salud en la planificación, evaluación de resultados, } \\
\text { seguridad del paciente y mejora de la calidad. }\end{array}$ \\
\hline Formación de los profesionales es esta área del conocimiento en beneficio de los pacientes. \\
\hline $\begin{array}{l}\text { Participación de los pacientes en el diseño, implementación y evaluación de los servicios } \\
\text { sanitarios. }\end{array}$ \\
\hline Análisis de las necesidades de los diferentes grupos de población y nivel de alfabetización. \\
\hline $\begin{array}{l}\text { Utilización de las estrategias de la alfabetización en salud en la mejora de la comunicación } \\
\text { interpersonal. }\end{array}$ \\
\hline Proporcionar facilidad de acceso a la información y a los servicios de salud. \\
\hline Diseño y elaboración de materiales de calidad y fáciles de entender. \\
\hline Detectar y actúar en poblaciones de riesgo elevado de padecer bajas competencias en salud. \\
\hline \begin{tabular}{l} 
Comunicar de manera clara y accesible la información sobre los servicios que ofrece. \\
\hline
\end{tabular} \\
\hline
\end{tabular}

Entre las estrategias necesarias para conseguir una alfabetización en salud adecuada de la población destacan la elaboración de programas de autocuidado de la propia enfermedad, el desarrollo de habilidades para establecer una relación de confianza entre pacientes y profesionales sanitarios, o la capacitación para actuar de forma adecuada en el manejo de la enfermedad o a través del sistema sanitario, entre otras acciones.

Además, la investigación de la alfabetización en salud en los sistemas sanitarios constituye un punto clave a tener en cuenta, por ser el ámbito de referencia para el seguimiento de las personas afectadas por una enfermedad crónica, en las cuales el paciente y el familiar van a tener que tomar numerosas decisiones durante el transcurso de la misma. De este modo, desarrollar estrategias para conocer el nivel de alfabetización del paciente, diseñar mensajes informativos comprensibles y canales que faciliten el acceso a la información, así como valorar la efectividad de las estrategias desarrolladas, puede contribuir a crear un contexto donde el intercambio comunicativo sea más rico y donde se produzca una mejora en el manejo de las enfermedades. 
Por otro lado, también los sistemas sanitarios y sus instituciones han de incorporar los elementos clave de la alfabetización en salud en sus agendas con el objetivo de que el paciente realmente ocupe el centro en el sistema. Así, por ejemplo, se desarrolló el plan de acción para mejorar la alfabetización sanitaria en Estados Unidos, bajo los principios de que toda persona tiene derecho a recibir información de calidad sobre su salud que le ayude a tomar decisiones informadas y que los servicios sanitarios se han de facilitar de manera que sean beneficiosos para la salud y la calidad de vida de la población (USDHHS, 2010). La Tabla 2 presenta algunas de las estrategias diseñadas para mejorar el nivel de alfabetización sanitaria de la población.

Tabla 2. Estrategias para mejorar el nivel de alfabetización sanitaria. Fuente: Adaptado de USDHHS (2010).

ESTRATEGIAS DE MEJORA

Desarrollar y hacer difusión de información de calidad sobre seguridad del paciente, que se entienda bien y sea rigurosa y accesible.

Potenciar cambios en el sistema sanitario que mejoren la información al paciente, la comunicación, la participación en la toma de decisiones y el acceso a los servicios sanitarios.

Incorporar información rigurosa y basada en la evidencia científica sobre salud y apropiada para el desarrollo en la educación infantil y hasta el nivel universitario.

Apoyar los esfuerzos de la comunidad para proporcionar programas formativos a personas adultas sobre salud y servicios sanitarios.

Promover partenariados, desarrollar guías de buenas prácticas y cambiar políticas que no favorezcan la mejora de la alfabetización en salud.

Favorecer la investigación sobre el desarrollo, la implementación y la evaluación de diferentes acciones para la mejora de la alfabetización en salud.

Aumentar la difusión y el uso de las intervenciones en alfabetización en salud basadas en la evidencia científica.

Las características del entorno de los centros sanitarios y las organizaciones determinan, por lo tanto, la experiencia de los pacientes que en ellos se tratan. Revisar estas características bajo el prisma de la alfabetización en salud ayudará a las instituciones y a sus profesionales a detectar las estrategias de mejora más efectivas para sus pacientes (Rudd et al., 2019). Relacionado con las características que han de tener de los centros sanitarios para favorecer estos aspectos, diferentes estudios han valorado las características arquitectónicas de los edificios (hospitales y centros de salud), su señalización, iluminación, navegabilidad o disposición para que las personas puedan transcurrir por sus espacios con facilidad (Groene y Rudd, 2011).

En otros casos, se ha puesto en evidencia que algunas de las barreras que encuentran los pacientes al intentar moverse por las organizaciones sanitarias son comunes. Tal es el caso de problemas en el acceso y la navegación por determinadas páginas web, interrupciones telefónicas durante la visita médica, escasez de indicaciones para encontrar un departamento o servicio, mapas de situación complejos o folletos informativos difíciles de entender, entre otras características que dificultan el acceso de los pacientes a la información y a la atención que necesitan (Rudd et al., 2013). 
Es necesario, por lo tanto, valorar cómo las organizaciones y los sistemas sanitarios, en general, tienen en cuenta las necesidades de los pacientes y facilitan su acceso y navegación por el sistema. De esta forma, los centros sanitarios llegarían a integrar la alfabetización en salud entre sus prioridades y como parte de su plan de acción conjuntamente con el paciente (Navarro Rubio et al., 2016).

\section{CONCLUSIONES Y POSIBLES ACTUACIONES}

El paciente en la actualidad es una persona con un mayor nivel de educación y acceso a la información que los pacientes de épocas anteriores. Esta situación conduce a un papel activo del paciente en el proceso de toma de decisiones de salud. También implica un cambio en la relación y la comunicación entre profesional y paciente.

La participación del paciente en la toma de decisiones puede realizarse a nivel individual, en el manejo de la enfermedad o contribuyendo a la mejora de los servicios de salud. Además, el paciente también puede participar representando a otros pacientes y abogando en la defensa de sus derechos. Existen diferentes niveles de participación del paciente en la toma de decisiones que van desde tener información de buena calidad o responder encuestas hasta colaborar e involucrarse en acciones de mejora continua de la calidad. Además, el empoderamiento del paciente significa que la persona, con información y experiencia, se siente segura para tomar decisiones informadas.

La formación es un tema clave para empoderar a los pacientes y participar en el proceso de toma de decisiones. La educación del paciente también ha evolucionado con el tiempo, desde las primeras experiencias del programa de pacientes expertos. Hoy en día, existe una gran variedad de acciones y programas de formación para pacientes y profesionales sobre cómo empoderar al paciente en el proceso de toma de decisiones en salud.

Por lo tanto, teniendo en cuenta los diferentes niveles de gestión en las organizaciones sanitarias, a nivel micro, la relación que se establece entre el profesional y el paciente, así como la formación del paciente y sus competencias y habilidades en alfabetización en salud, van a ser muy importantes. Todo ello unido a las características y formación del profesional sanitario de manera que facilite este rol del paciente más activo.

En mesogestión, a nivel de las organizaciones sanitarias, es necesario facilitar el acceso y la navegación del paciente en la institución, así como que pueda utilizar la información adecuada para hacer buen uso de su salud y de los servicios sanitarios.

A nivel macro, en las administraciones y sistemas de salud de los gobiernos, es necesario establecer políticas y planes de acción que integren al paciente en el proceso de toma de decisiones. En este sentido, sería necesario apoyar la realización de programas que hagan aumentar el nivel de alfabetización en salud de la población en futuras generaciones y a través de los diferentes niveles educativos. Es, por lo tanto, imprescindible, el esfuerzo conjunto de áreas de la medicina, la salud pública, la educación y la comunicación (Rudd et al., 2013).

Así pues, y aunque es necesario que el sector educativo de la sociedad mejore con sus programas el nivel de alfabetización en salud de la población, es necesario empezar a actuar ya en el sector sanitario eliminando las barrearas que impiden a los pacientes acceder a información de calidad, a los servicios y a su cuidado.

A la vez, y mientras el sector sanitario refuerza estas medidas, se debe favorecer la investigación para conocer el impacto de la educación del paciente sobre el estado de salud y el uso de los servicios de salud, así como sobre las formas de evaluar y medir estos resultados y 
se han de asignar recursos institucionales que favorezcan la participación real del paciente informado en la toma de decisiones (Rudd et al., 2013).

\section{REFERENCIAS BIBLIOGRÁFICAS}

Berkman, N. D., Sheridan, S. L., Donahue, K. E. et al. (2011). Low health literacy and health outcomes: An updated systematic review. Annals of Internal Medicine, 155(2), 97-107. https://doi.org/10.7326/0003-4819-155-2-201107190-00005

Brach, C., Keller, D., Hernández, L. M. et al. (2012) Ten attributes of health literate health care organizations. Institute of Medicine of the National Academies.

Brega, A. G., Hamer, M. K., Albright, K. et al. (2019). Organizational health literacy: Quality improvement measures with expert consensus. Health Literacy Research and Practice, 3(2), e127-e146. https://doi.org/10.3928/24748307-20190503-01

Davis, T. C., Long, S. W., Jackson, R. H. et al. (1993). Rapid estimate of adult literacy in medicine: A shortened screening instrument. Family Medicine, (25), 391-395.

González de Paz, L., Gálvez Hernández, P. y Navarro Rubio, M. D. (2019). Development and psychometric study of a simple instrument to assess patient communication and comprehension skills: the AsCkS. Family Practice, 4(1), 568-573. https://doi.org/10.1093/fampra/cmz087

Groene, R. O. y Rudd, R. E. (2011). Results of a feasibility study to assess the health literacy environment: Navigation, written, and oral communication in ten hospitals in Catalonia, Spain. JCIH, 4(4), 227 237. https://doi.org/10.1179/1753807611Y.0000000005

Halvorsen, K., Dihle, A., Hansen, C. et al. (2020). Empowerment in health care: A thematic synthesis and critical discussion of concept analyses of empowerment. Patient Education and Counseling, 103(7), 1263-1271. https://doi.org/10.1016/i.pec.2020.02.017

Haun, J., Luther. S., Dodd, V. et al. (2012). Measurement variation across health literacy assessments: Implications for assessment selection in research and practice. Journal of Health Communication, 17(Issue sup3), 141-159. https://doi.org/10.1080/10810730.2012.712615

Heijmans, M., Waverijn, G., Rademakers, J. et al. (2015). Functional, communicative and critical health literacy of chronic disease patients and their importance for self-management. Patient Education and Counseling, 98(1), 41-48. https://doi.org/10.1016/i.pec.2014.10.006

Howard. D. H., Gazmararian, J. y Parker, R. (2005). The impact of low health literacy on the medical costs of Medicare managed care enrollees. American Journal of Medicine, 118(4), 371-377. https://doi.org/10.1016/j.amjmed.2005.01.010

Kimble, J. (2002). The elements of plain language. Michigan Bar Journal, (81), 44-45.

Koch-Weser, S., DeJong, W. y Rudd, R. E. (2009). Medical word use in clinical encounters. Health Expectations, 12(4), 371-382. https://doi.org/10.1111/i.1369-7625.2009.00555.x

Kutner, M., Greenberg, E., Jin, Y. et al. (2006). The health literacy of America's adults: results from the 2003 National Assessment of Adult Literacy. National Center for Education Statistics, US Department of Education.

Lai, A. Y., Ishikawa, H., Kiuchi, T. et al. (2013). Communicative and critical health literacy, and selfmanagement behaviors in end-stage renal disease patients with diabetes on hemodialysis. Patient Education and Counseling, 91(2), 221-227. https://doi.org/10.1016/j.pec.2012.12.018

Montori, V. (2017). ¿Qué es la toma de decisiones compartida (y qué no lo es)? XPac and Health Communication, (1).

Navarro Rubio, M. D., Gabriele Muñiz, G. y Jovell Fernández, A. J. (2008). Los derechos del paciente en perspectiva. Atención Primaria, 40(7), 367-369. https://doi.org/10.1157/13124131

Navarro Rubio, M. D. (2014). Pacientes implicados: Participación del paciente en la toma de decisiones. Papeles de economía española, (142), 85-91. https://www.funcas.es/wpcontent/uploads/Migracion/Articulos/FUNCAS PEE/142art11.pdf

Navarro Rubio, M. D., Rudd, R., Rosenfeld, L. et al. (2016). Health literacy: Implications for the health system. Medicina Clínica, 147(4), 171-175. https://doi.org/10.1016/j.medcle.2016.09.020

Navarro Rubio, M. D., Gálvez Hernández, P. y Virumbrales Cancio, M. (2018). Ciudadanos y Pacientes. En A. Martín Zurro y G. Jodar Solá (Eds.), Atención familiar y salud comunitaria (pp. 31-45). Elsevier. 
Navarro Rubio, M. D., Gálvez, P., Vázquez, J. et al. (2019). Valoración de las competencias en salud de los pacientes: Instrumento VACS. Journal of Healthcare Quality Research, 34(4), 193-200. https://doi.org/10.1016/i.jhqr.2019.04.005

Neilsen-Bohlman, L., Panzer, A. M. y Kindig, D. A. (2004). Health literacy: A prescription to end confusion. National Academies Press. https://doi.org/10.17226/10883

Nutbeam, D. (2000). Health literacy as a public health goal: a challenge for contemporary health education and communication strategies into the 21st century. Health Promotion International, (15), 259-267.

OCDE (2013). Evaluación de competencias de adultos. Recuperado de http://skills.oecd.org

Paasche-Orlow, M. K., Schillinger, D., Weiss, B. D. et al. (2018). Health literacy and power. Health Literacy Research and Practice, 2(3), e132-e133. https://doi.org/10.3928/24748307-20180629-01

Parker, R. M., Baker, D. W., Williams, M. V. et al. (1995). The test of functional health literacy in adults: a new instrument for measuring patients' literacy skills. Journal of General Internal Medicine, (10), 537-541. https://doi.org/10.1007/BF02640361

Patak, L., Wilson-Stronks, A., Costello, J. et al. (2009). Improving patient-provider communication: A call $\begin{array}{ll}\text { to action. Journal of Nursing Administration, 39(9), 372-376. } & \text {, }\end{array}$ https://doi.org/10.1097/NNA.0b013e3181b414ca

Rudd, R. E. (2010). Improving Americans' health literacy. New England Journal of Medicine, (363), 22832285. https://doi.org/10.1056/NEJMp1008755

Rudd, R. E., Groene, O. R. y Navarro-Rubio, M. D. (2013). On health literacy and health outcomes: Background, impact, and future directions. Revista de Calidad Asistencial, 28(3), 188-192. https://doi.org/10.1016/j.cali.2013.03.003

Rudd, R. E., Oelschleger, S., Grabeel, K. L. et al. (2019). The health literacy environment of hospitals and health centers. Assessment tool. Harvard T.H. Chan School of Public Health.

Sørensen, K. et al. (2011). Literature review of health literacy for the development of a theoretical model. HLS-EU Consortium.

Speros, C. I. (2011). Promoting health literacy: A nursing imperative. Nurs Clin North America, 46(3), 321-333. https://doi.org/10.1016/j.cnur.2011.05.007

Stiggelbout, A. M., Van der Weijden, T., De Wit, M. P. et al. (2012). Shared decision making: really putting patients at the centre of healthcare. British Medical Journal, (344), e256. https://doi.org/10.1136/bmj.e256

USDHHS (U.S. Department of Health and Human Service, Office of Disease Prevention and Health Promotion) (2010). National Action Plan to improve health literacy. USDHHS.

Weiss, B. D. (2007). Health literacy and patient safety: Help patients understand. American Medical Association and AMA Foundation.

Wolf, M. S., Wilson, E. A., Rapp, D. N. et al. (2009). Literacy and learning in healthcare. Pediatrics, 124(is3), S275-S281. https://doi.org/10.1542/peds.2009-1162C

Yen, P. H. y Leasure, R. (2019). Use and effectiveness of the teach-back method in patient education and health outcomes. Fed Practice, (36), 284-289. 